\title{
The Future of Theory: should social protection board the big data train?
}

\section{Cedric Waterschoot ${ }^{1}$}

KNAW Meertens Instituut, Amsterdam, The Netherlands

Applications of big data have been surging as of late, and the field of public policy does not stand on the sideline while this dramatic wave of new technologies makes its way across the disciplines. However, theory-driven fields may experience radical change, as data fundamentalists claim the end of theory will come due to the nature and practicality of big data. In this paper, the position of social protection is examined with regard to the effects of the already observed shift towards such computational methods. I argue that such dramatic end of theory will not come for social protection policy, as the specialists and theorists take up the role of interpreter of data, performing the needed task of translating the vast collection of information into a useable collection or result. Vital in this position is the contact with the political economy, a task impossible to result in fruitful outcome without the interpreter. To strengthen this position in regard to social protection and big data, two examples are outlined: 'Citizen Based Analytics' in New-Zealand and the Big Data Quality Task Team of the UNECE.

Keywords: Social protection, big data, public policy, data fundamentalism

\footnotetext{
${ }^{1}$ cedric.waterschoot@meertens.knaw.nl
} 
"Change the instruments, and you will change the entire social theory that goes with them"

Bruno Latour 2009

\section{Introduction}

Without a doubt, the big data train has passed a station in everyone's life. Whether through social media, online shopping or other services using databases and algorithms, every individual has contributed to a vast collection of enormous datasets. This stream of behavioural data coming straight from the source is seen as an opportunity by many. Others recognize a threat, citing issues ranging from privacy and asymmetrical information (Lepri, Staiano, Sangokoya, Letouzé, \& Oliver, 2017) to methodological roadblocks (Boyd \& Crawford, 2012). It is vital to analyse what this means for the future of academia and research, particularly those fields who have not fully surrendered to the calls of (pseudo-) objectivity of quantification. These disciplines, which include social protection to a certain extent, still rely on qualitative data (often in combination with quantitative analysis). The quantitative call will only become stronger with the introduction of so-called 'Big Data Techniques'. As Bruno Latour states in the opening sentence of his essay: "Numbers, numbers, numbers. Sociology has been obsessed by the goal of becoming a quantitative science" (Latour, 2003).

Will social protection as a discipline and practical implementation and governance structures surrender to what data fundamentalists call the 'End Of Theory' (Anderson, 2008)? Will the data speak for itself in so far that human interpretation becomes complementary, maybe even obsolete? This essay will address the beforementioned questions by exploring the rise of Big Data in regard to social protection and targeted programmes in particular. The following statement will be presented and analysed: 'Big data will not lead to the 'end of theory' in targeted social protection. Human Interpretation and evaluation will be needed as big data will not speak for itself.' First of all, the two arguments will be presented along with an accurate definition of the Big Data phenomenon. Secondly, the role of the social protectionist will be suggested as the interpreter of Big Data in order to craft targeted social policies by countering arguments made by the data fundamentalists.

\section{Big Data, Big Debate}

The term 'Big Data' is omnipresent in both the scientific landscape and the wider population. But what does this phenomenon actually entail? Many have their own definition. Some argue that there is no one single meaning of big data (Boellstorff, 2013). Others simply state that it encompasses datasets so big supercomputers are needed (Manovich, 2013). Kate Crawford, however, proposes the concept of Big Data to include the computational techniques and analytical methods, datasets and what she calls the mythology stating that "large data sets offer a higher form of intelligence and knowledge that can generate insights that were previously impossible, with the aura of truth, objectivity, and accuracy" (Boyd \& Crawford, 2012). From here on in this paper, Big Data will mean the latter: a combination of 
extremely big datasets, analytical tools to adequately dissect them and the stern belief that these will return favourable and applicable results. However, this infallible conviction of unmatched predictive and descriptive capacity is not shared by all scholars working on Big Data. The next section outlines this divide.

\section{Data Fundamentalism}

Those who advocate that the end station of the Big Data train proclaims the 'End of Theory' have been christened the data fundamentalists (Crawford, Milner, \& Gray, 2014). These scholars and advocates put forward the idea that when the datasets grow this large and entail such an abundance of precise, behaviour information, the data will speak for itself, without the need of theoretical assumptions, frameworks and theorists themselves. As Chris Anderson, who first wrote about the 'End of Theory', proclaims himself: "with enough data, the numbers speak for themselves" (Anderson, 2008).

The overarching goal is reaching a probabilistic universe in which behaviour is predicted through the vast collection of data (Pentland, Haas, \& George, 2014). Pentland and his colleagues claim that through Big Data, we reach a society better equipped to prevent corruption, political distortion and financial crashes. In the case of scientific disciplines and academia, of which social protection is part of, the scientist as a person will be less needed. Anderson himself proclaimed that this shift occurs due to the fact that "statistical algorithms find patterns where science cannot" (Anderson, 2008). According to this line of thought, the focus of analysis will shift as well. Correlation in these large-scale datasets will lead the way. Causation focussed research will fade away and be replaced by the "precise-enough" correlational analysis (Mayer-Schönberger \& Cukier, 2013). Mayer-Schönberger goes even a step further with his shift towards correlation over causation, stating that the smaller focus on 'exactitude' means that our data will more adequately represent the messiness and complexity of nature. What does this mean for social protection? One can ask themselves the following question: is it good enough to know that something (e.g. poverty) is happening or do we need to focus on why it exists? Data fundamentalists claim the former, that in most cases it is sufficient to know poverty exists and who are poor (Mayer-Schönberger \& Cukier, 2013). This line of thinking results in a major shift in research, i.e. a change from hypothesis-testing methodologies towards hypothesis-generating analysis.

To sum up, data fundamentalists claim that if Big Data research is based on sound statistical manipulation and bottom-up induction, no theory is required. Furthermore, if no theory is needed, there is no place for a theorist either. What use has a social protection specialist if the data has already informed governments what each and every citizen's capabilities require? Can they not simply provide that what is exactly needed and combat poverty in an efficient manner? The Big Data train can transport government officials exactly where they need to go. Administrations simply need to buy a ticket. 


\section{Countermovement}

There is a growing sentiment that, while accepting Big Data as a positive opportunity to add to the research tool bench, it is of utter most importance that one assesses the data critically and, most importantly, to not accept that Big Data will side line all other research and be the future of analysis. Among others, one of the most prominent voices is Kate Crawford. She and her colleagues advocate to strictly outline concerns, e.g. privacy and other ethical considerations, and to firmly reject the mythology around the phenomenon (Boyd \& Crawford, 2012; Crawford et al., 2014). Before addressing the specific considerations in regard to social protection theory and policy, it is vital to acknowledge that Big Data is still kept in silos, outside the reach of most of us (Mazzocchi, 2015). Crawford warns that, if the current ecology of Big Data is not reformed, it will lead to a new form of inequality. This digital inequality divides the "Big Data rich from the Big Data poor" (Boyd \& Crawford, 2012). Policy makers need to realize that it is within their reach to craft this ecosystem anew and avoid this polarization of control over Big Data and inevitably halt digital inequality.

In the case of social protection, two factors come to mind that need to be addressed in response to the 'End of Theory' claims. First of all, from a theoretical perspective on data collection and analysis, hidden biases have the capability to distort academic work on social protection interventions. Furthermore, addressing shortcomings in representativity and quality of data is detrimental in designing effective policy and most of all, crafting secure targeting mechanisms. The concept of hidden biases have been presented by Crawford (Boyd \& Crawford, 2012). These authors demand researchers to pose some of the following questions about their dataset: (1) Are certain demographics excluded due to the digital nature? (2) Are irrelevant factors overemphasised in the sample (e.g. massive amount of cell phone use in irrelevant geographical areas)? (3) Does this dataset cast a shadow over some individuals? (Boyd \& Crawford, 2012). For targeted social protection policies, these questions are of utter most importance. Crafting policy to address older age groups is not possible if these are not in the sample due to the fact that this demographic is underrepresented on social media. Furthermore, if social protection exists to combat and minimize inequalities around the globe, the usage of inadequate datasets may amplify existing inequalities and ethical problems (Gillingham \& Graham, 2017). This effect only strengthens if the digital inequality overlaps with already existing inequalities. If Big Data analysis is to be used in order to craft targeted social protection policies, these hidden biases need to be account for. This form of accountability is omitted if one allows to let the numbers speak for themselves. Social data specialists are required to assess this stage in the research cycle.

Secondly, identifying social problems and potential policies to address these issues is by definition a political, and therefore contested, process (Henman, 2004). Data alone is incapable of navigating a complex ecosystem called the 'political economy'. Alongside political debate comes a second discussion on the usage of statistical reasoning and most importantly, how to measure a social phenomenon like inequalities or poverty (Desrosières, 2002). These discussions entail arguments on who or what the target ought to be and how to select indicators. All of the beforementioned issues are 
inherently influenced by political factors (Henman \& Adler, 2005). Data alone can never be capable of crafting policy that undergoes such a complex railway of economic, political and even philosophical questions. Once again, a specialist is needed in order to systematically address the issues raised above and to navigate the turbulent waters of the political economy.

As the data increases in size, so does the potential of hidden biases. As the role of Big Data strengthens in policy making, so does the contact with the political economy. Therefore, a social protection specialist is needed to take on the role of interpreter of data.

\section{The Interpreter}

As mentioned before, the evolution and momentum of Big Data will not lead to the 'End of Theory' as proposed by data fundamentalists, due to the fact that numbers alone fall short of winning political debates and often fall prey to hidden biases which might lead to the strengthening of what social protection aims to reduce. The following paragraphs will propose the social protection specialist's role as interpreter and shepherd of Big Data with the goal of countering some of the claims made by data fundamentalists that might not hold up in the realm of social protection.

To begin with, it is vital to recognize that political choices are in need of argumentation that bottom-up induction and correlation cannot provide. It might be the case that for academic fields that simply need result presentation, Big Data leads to a minimization of the theorist's role. However, social protection policy requires a prolonged interaction with political stakeholders and successful implementation relies on positive outcomes in debates. As was mentioned before, the choosing of indicators, target groups and definitions of social problems are fully reliant on social protection specialists, even in the presence of strong statistical evidence procured from correlations out of Big Data. Otherwise, the numbers will fall without a voice.

Secondly, the interpretative role of the social protectionist entails the safekeeping of the dataset against hidden biases. Shortcomings need to be addressed and, if possible, account for. Behaviour prediction based on a vast collection of data still leads to biased results if the dataset is unrepresentative. Correlations stemming out of these flawed samples are unusable for policies. Furthermore, data collected through politically fuelled channels cause the sample not to be neutral. The 'correlation sufficiency' argument made by data fundamentalists do not hold for these kinds of datasets due to the direct impact on policy. Additionally, it is vital that the role of the social protectionist includes the detection of deindividualization. This form of overgeneralization and attribution of relevant characteristics on individuals who may not possess them undoubtably leads to flawed targeting of social protection programmes. Deindividualization has been seen as a potential consequence of unsupervised usage of computational methods for social policy.

Last but not least, the argumentation put forward by data fundamentalists states that the researching the ' $w h y$ ' will be put to rest in the shadow of the fact that it does occur. For social protection, however, the causes of poverty and forms of inequality are on the forefront and cannot be side-lined by 
other research goals. In this case, the numbers may only speak for themselves to a certain extent and are in dire need to be supplemented by additional (qualitative) research and interpretation. The why patterns will always be placed under the wing of the analysis (Gillingham \& Graham, 2017).

To sum up, the social protectionist as interpreter and gatekeeper will be a vital companion of Big Data, which will inevitably lead to new opportunities in formulation and evaluation of social policy. This role includes theory, which is necessary to achieve policies targeted at those in need, due to the fact that the numbers do not speak for themselves, especially in heated political debate. Furthermore, studying underlying symptoms of poverty and inequality are extremely important in crafting rigid policy. The function of the specialist adapts to new circumstances but remains vital as ever before.

\section{On Board}

To substantiate the argumentation as to why Big Data will not lead to the 'End of Theory' in the field of social protection, a closer look at projects that have been put to practice can help.

An example of Big Data informing social policy exists in New Zealand. The so-called 'CitizenBased Analytics' is a data driven approach with the goal of optimizing social investments. As is written by the Chief Science Advisor, the goal is " [...] to allow policymakers to decide which interventions, delivered to which individuals, at what stage in their life-course, will do the most to boost resilience later in life" (Gluckman, 2017). Important for the discussion in this paper is the role that is outlined in this Big Data project for the data expert and social protectionist. A clear function for individuals in term of data curation, management and analysis has been included, as to safeguard the outcomes from the pitfalls in Big Data usage that have been outlined in the previous chapters. The Chief Science Advisor goes even one step further by recommending universities to train students in data hygiene and interpretation skills, as they will be needed in order to cope with the Big Data avalanche. Without these social protection experts, the advancements made from these new techniques may not reach the political debate table. Furthermore, it is stressed in the Citizen-Based Analytics system that it will not replace the need for qualitative and traditional policy analysis methods (Gluckman, 2017). Argued is the vital requirement of mixed methods and purely qualitative work that exposes the exact themes that Big Data analytics can dig into.

Additionally, the Big Data Quality Task Team of the UNECE has produced a framework to assess the quality of this distinct strain of research (UNECE/HLG, 2014). This particular framework stresses the importance of additional analysis of the obtained Big Data and its sources in a thorough manner. The role of experts to investigate the obtained samples is vital to determine the validity and reliability. Critical reflection by National Statistical Offices or other specialists allows the correct usage of such data.

To conclude, these two examples highlight the practical feasibility and need for specialists, in this case social protection theorists, to complement Big Data use in policy making. Theory and experts still play a vital role in Big Data contexts. 


\section{Conclusion}

To sum up, argumentation in favour and against the so-called 'End of Theory' in social sciences has been presented. The position against this development and in favour of the responsibility of the social protectionist as interpreter of data is outlined by countering the argument that numbers will speak for themselves. Furthermore, without the expert to safeguard large datasets from hidden biases and politically influenced sources, the correlations will be flawed, and future policy will be both ineffective and wrongly targeted. Lastly, underlying symptoms would be left out if Big Data is to operate without the theorist. However, it is important to recognize the immense opportunities that social protection receives from the advancements in Big Data. Future policies will benefit without a doubt from these types of analysis. Therefore, it is of utter most importance that from the beginning on it is accepted that numbers alone are voiceless without adequate interpretation of trained specialists.

The social protectionist ought to be the flashlight carrying guide in an otherwise unlit labyrinth of Big Data caves. The right path might have been chosen without guidance either way, but who knows how many wrong turns you take. For social protection policy, incorrect paths may cause dire consequences for those in need of support. Therefore, it is vital that Big Data analyses for social protection are always accompanied by an interpreter with expertise in both social protection and data analysis theories. Big Data will not lead to the 'End of social protection Theory'. It is merely the beginning of a new responsibility of trained experts. The theories that have been crafted and perfected over the years might turn out to be the exact addition the Big Data train needs in order to reach the end station. 


\section{References}

Anderson, C. (2008). The End of Theory: The Data Deluge Makes the Scientific Method Obsolete. Wired. Retrieved from https://www.wired.com/2008/06/pb-theory/

Boellstorff, T. (2013). Making big data, in theory. First Monday.

Boyd, D., \& Crawford, K. (2012). Critical questions for big data: Provocations for a cultural, technological, and scholarly phenomenon. Information Communication and Society, 15(5), 662679. https://doi.org/10.1080/1369118X.2012.678878

Crawford, K., Milner, K., \& Gray, M. (2014). Critiquing Big Data: Politics, Ethics, Epistemology. International Journal of Communication, 1663-1672. https://doi.org/10.1080/10511259900084631

Desrosières, A. (2002). The Politics of Large Numbers.

Gillingham, P., \& Graham, T. (2017). Big Data in Social Welfare: The Development of a Critical Perspective on Social Work's Latest “Electronic Turn.” Australian Social Work, 70(2), 135-147. https://doi.org/10.1080/0312407X.2015.1134606

Gluckman, P. (2017). Using evidence to inform social policy: The role of citizen-based analytics, (505), $1-25$.

Henman, P. (2004). Targeted!: Population Segmentation, Electronic Surveillance and Governing the Unemployed in Australia.

Henman, P., \& Adler, M. (2005). Computerizing the welfare state.

Latour, B. (2003). 9 Tarde's idea of quantification *.

Lepri, B., Staiano, J., Sangokoya, D., Letouzé, E., \& Oliver, N. (2017). The Tyranny of Data? The Bright and Dark Sides of Data-Driven Decision-Making for Social Good, 3-24. https://doi.org/10.1007/978-3-319-54024-5_1

Mayer-Schönberger, V., \& Cukier, K. (2013). Big Data: A Revolution that Will Transform how We Live, Work, and Think.

Mazzocchi, F. (2015). Could Big Data be the end of theory in science? EMBO Reports.

Pentland, A., Haas, M., \& George, G. (2014). Big Data and Management.

UNECE/HLG. (2014). A Suggested Framework for the Quality of Big Data: Deliverables of the UNECE Big Data Quality Task Team. 